TITLE:

\title{
Second- and higher-order quasinormal modes in binary black-hole mergers
}

$\operatorname{AUTHOR}(S):$

loka, K; Nakano, H

CITATION:

loka, K ... [et al]. Second- and higher-order quasinormal modes in binary black-hole mergers. PHYSICAL REVIEW D 2007, 76(6): 061503.

ISSUE DATE:

2007-09

URL:

http://hdl.handle.net/2433/49823

RIGHT:

Copyright 2007 American Physical Society 
PHYSICAL REVIEW D 76, 061503(R) (2007)

\title{
Second- and higher-order quasinormal modes in binary black-hole mergers
}

\author{
Kunihito Ioka ${ }^{1}$ and Hiroyuki Nakano ${ }^{2}$ \\ ${ }^{1}$ Department of Physics, Kyoto University, Kyoto 606-8502, Japan \\ ${ }^{2}$ Center for Computational Relativity and Gravitation, School of Mathematical Sciences, Rochester Institute of Technology, \\ Rochester, New York 14623, USA
}

(Received 25 April 2007; published 13 September 2007)

\begin{abstract}
Black-hole $(\mathrm{BH})$ oscillations known as quasinormal modes (QNMs) are one of the most important gravitational wave $(\mathrm{GW})$ sources. We propose that higher perturbative order of QNMs, generated by nonlinear gravitational interaction near the BHs, are detectable and worth searching for in observations and simulations of binary $\mathrm{BH}$ mergers. We calculate the metric perturbations to second order and explicitly regularize the master equation at the horizon and spatial infinity. We find that the second-order QNMs have frequencies twice the first-order ones and the GW amplitude is up to $\sim 10 \%$ of that of the firstorder one. The QNM frequency would also shift blueward up to $\sim 1 \%$. This provides a new test of general relativity as well as a possible distance indicator.
\end{abstract}

DOI: 10.1103/PhysRevD.76.061503

\section{INTRODUCTION}

Direct detections of gravitational waves (GWs) will become a reality in the near future with current and future detectors, such as LIGO, LISA, and DECIGO/BBO [1]. GWs can not only provide a test of general relativity but also open a new window on the universe.

One of the most important GW sources are the quasinormal modes (QNMs) of black holes (BHs) [2]. QNMs are oscillations of the $\mathrm{BH}$ metric perturbations, and they are damped by emitting GWs. QNM frequencies are complex with the real part representing the oscillation frequency and the imaginary part representing the damping. By observing the QNM frequencies, one can determine the mass and angular momentum of spinning BHs.

The most promising sources that excite QNMs are binary BH mergers. For these events QNMs will be detected with high signal-to-noise ratio (SNR) (e.g., SNR $\sim 10^{5}$ for $\sim 10^{8} M_{\odot}$ BH mergers at $\sim 1$ Gpc by LISA) [3] since a large fraction of the energy $(E \sim 1 \% \times M$ for equal-mass mergers with a total mass $M$ ) goes into GWs. The merger rate is also estimated to be large enough $[4,5]$. In numerical relativity there have been recent breakthroughs for calculating the entire phase of binary BH mergers [6]. The result is that the $\ell=2, m=2 \mathrm{QNM}$ is dominant, carrying away $\sim 1 \%$ of the initial rest mass of the system [7].

In this paper we show that the higher-order QNMs are also prominent in binary $\mathrm{BH}$ mergers and they are interesting to search for in the observations and simulations. Here the "higher-order" is with respect to the metric perturbations. Although the dimensionless amplitude of the metric perturbations are negligibly small when we observe them at detectors, they are relatively large near the $\mathrm{BH}$, up to

$$
\psi^{(1)} / M \sim(E / M)^{1 / 2} \sim 10 \%
$$

for QNMs with energy $E / M \sim 1 \%$ [see Eq. (16) with $\omega \sim$ $M^{-1} . \psi^{(1)}$ denotes a wave function to be discussed later]. Hence the generated second-order perturbations would
PACS numbers: 04.30.-w, 04.70.Bw, 95.55.Ym, 95.85.Sz

have the amplitude $\sim 10 \%$ of the first-order ones, which may be detectable for high SNR events.

Higher-order QNMs are essentially analogous to the anharmonic oscillations discussed by Landau and Lifshitz in Mechanics [8]. In general, an oscillation with small amplitude $x$ is described by an equation, $\ddot{x}+\omega^{2} x=$ 0 , which gives the first-order solution $x=a \cos (\omega t+\phi)$. Here $a$ and $\phi$ are integration constants. Including the second-order term with respect to the amplitude $\sim x^{2}$, the equation becomes

$$
\ddot{x}+\omega^{2} x=-\alpha x^{2} .
$$

With the right-hand side as a source term, we obtain a successive solution $x=a \cos (\omega t+\phi)+x^{(2)}$, where

$$
x^{(2)}=\frac{\alpha a^{2}}{6 \omega^{2}} \cos 2 \omega t-\frac{\alpha a^{2}}{2 \omega^{2}} \propto a^{2} .
$$

Here an oscillation arises with a frequency $2 \omega$. The important point is that the second-order oscillation $x^{(2)}$ is driven by the first-order one and thereby always exists.

Our result is that the second-order QNMs would also have frequencies twice the first-order ones $\omega^{(2)}=2 \omega^{(1)}$ and amplitude up to $\sim 10 \%$ of the first-order ones. Since higher-order QNMs always exist, we may test the nonlinearity of general relativity. The purpose of this paper is to outline the calculations of the second-order QNMs for the Schwarzschild BH and clarify the order-of-magnitude estimates. More details will be given in the forthcoming papers. We use the units $c=G=1$, and arbitrarily set $M=1$ which we can always recover, if necessary.

Although this paper is the first to study second-order QNMs, the second-order analysis is pioneered by Tomita [9], and the $\ell=2, m=0$ case in the Schwarzschild spacetime is studied by Gleiser et al. [10]. It is also extended to cosmology [11] and the Kerr case [12]. 


\section{FIRST ORDER}

Let us consider the metric perturbations to second order,

$$
\tilde{g}_{\mu \nu}=g_{\mu \nu}+h_{\mu \nu}^{(1)}+h_{\mu \nu}^{(2)},
$$

where $g_{\mu \nu}$ is the Schwarzschild metric and the superscripts denote a perturbative order. Expanding the Einstein's vacuum equation we can obtain basic equations order by order $[10,13]$.

For the first order, we use the Regge-Wheeler-Zerilli formalism [14,15]. Separating angular variables with tensor harmonics of indices $(\ell, m)$, the equations decouple to the even (or polar) part with parity $(-1)^{\ell}$ under a transformation $(\theta, \phi) \rightarrow(\pi-\theta, \pi+\phi)$ and the odd (or axial) part with parity $(-1)^{\ell+1}$. Seven equations for the even parity part are reduced to a single Zerilli equation, and the other three equations for the odd parity part to a single Regge-Wheeler equation. The Zerilli equation is given by

$$
\begin{aligned}
& {\left[-\frac{\partial^{2}}{\partial t^{2}}+\frac{\partial^{2}}{\partial r_{*}^{2}}-V_{Z}(r)\right] \psi_{\ell m}^{(1)}(t, r)=0,} \\
& V_{Z}(r)=\left(1-\frac{2}{r}\right) \frac{2 \lambda^{2}(\lambda+1) r^{3}+6 \lambda^{2} r^{2}+18 \lambda r+18}{r^{3}(\lambda r+3)^{2}},
\end{aligned}
$$

where $r_{*}=r+2 \ln (r / 2-1)$ and $\lambda=(\ell-1)(\ell+2) / 2$. By Fourier transforming, $\psi_{\ell m}^{(1)}(t, r)=\int e^{-i \omega t} \psi_{\ell m \omega}^{(1)}(r) d \omega$, the Zerilli equation gives a one-dimensional scattering problem, which is familiar from quantum mechanics. The QNMs are obtained by imposing the boundary conditions with purely ingoing waves $\psi_{\ell m \omega}^{(1)} \sim e^{-i \omega r_{*}}$ at the horizon and purely outgoing waves $\psi_{\ell m \omega}^{(1)} \sim e^{i \omega r_{*}}$ at infinity. Such boundary conditions are satisfied at discrete QNM frequencies $\omega_{\ell m n}^{(1)}$ that are complex with the imaginary part representing the damping. There are an infinite number of QNMs for each harmonic $(\ell, m)$ [16].

All physical quantities for the first-order even parity part can be reconstructed from $\psi_{\ell m}^{(1)}$. If we define

$$
\psi_{\ell m}^{(1)}=\frac{r}{\lambda+1}\left[K_{\ell m}^{(1)}+\frac{r-2 M}{\lambda r+3 M}\left(H_{2 \ell m}^{(1)}-r \frac{\partial K_{\ell m}^{(1)}}{\partial r}\right)\right]
$$

in the Regge-Wheeler (RW) gauge according to [17,18], the $\mathrm{GW}$ power is given by

$$
\frac{d E^{(1)}}{d t}=\frac{1}{64 \pi} \sum_{\ell m} \frac{(\ell+2) !}{(\ell-2) !}\left|\frac{\partial}{\partial t} \psi_{\ell m}^{(1)}(t, r)\right|^{2} .
$$

Note that in the RW gauge the gauge freedom is completely fixed and the physical quantities can be expressed by the gauge invariant functions in simple differential forms.

In this paper we focus on the most dominant modes in binary $\mathrm{BH}$ mergers, i.e., the $\ell=2, m=2$ even parity mode for the first order [6] and its driving second-order mode (the $\ell=4, m=4$ even parity mode as shown be- low). Note that we have to specify $m$ since the degeneracy between $m$ breaks at the second order.

\section{SECOND ORDER}

For the second order, we also separate angular variables in terms of tensor harmonics. Instead of $\psi_{\ell m}^{(2)}$, we introduce a function

$$
\chi_{\ell m}^{(2)}=\frac{r-2 M}{\lambda r+3 M}\left[\frac{r^{2}}{r-2 M} \frac{\partial K_{\ell m}^{(2)}}{\partial t}-H_{1 \ell m}^{(2)}\right]
$$

in the RW gauge for convenience, which is essentially the time-derivative of $\psi_{\ell m}^{(2)}$ [10]. The first-order counterpart exactly satisfies $\chi_{\ell m}^{(1)}=\partial \psi_{\ell m}^{(1)} / \partial t$, and so the dimensions are $\psi_{\ell m}^{(i)} \sim O(M)$ and $\chi_{\ell m}^{(i)} \sim O\left(M^{0}\right)(i=1,2)$. The equations for the even parity part are reduced to the Zerilli equation with a source term,

$$
\left[-\frac{\partial^{2}}{\partial t^{2}}+\frac{\partial^{2}}{\partial r_{*}^{2}}-V_{Z}(r)\right] \chi_{\ell m}^{(2)}(t, r)=S_{\ell m}(t, r),
$$

where the source term $S_{\ell m}$ is quadratic in $\psi_{\ell m}^{(1)}$.

The most dominant second-order mode is the $\ell=4$, $m=4$ even parity one. This is because the dominant first-order mode is the $\ell=2, m=2$ even parity mode and hence $S_{\ell m}$ is dominated by the product $(\ell=2, m=$ $2) \times(\ell=2, m=2)$ in Eq. (9). This gives the $m=4$ mode that is $\ell=4$ and even parity. Note that what we are considering is the particular solution. Homogeneous solutions are just proportional to the first-order ones and are therefore trivial.

\section{REGULARIZATION}

Although it is straightforward to calculate the $\ell=4$, $m=4$ source term $S_{44}$ in terms of $\psi_{22}^{(1)}$, the raw source term does not behave well at infinity and is not suitable for calculations. We can find $S_{44} \sim O\left(r^{0}\right)$ at infinity by using the expansion

$$
\psi_{22}^{(1)}=\frac{1}{3} F_{I}^{\prime \prime}+\frac{1}{r} F_{I}^{\prime}+\frac{1}{r^{2}}\left(F_{I}-F_{I}^{\prime}\right)+O\left(r^{-3}\right),
$$

where $F_{I}=F_{I}\left(T_{-}\right)$is some function of $T_{-}=t-r_{*}$ and $F_{I}^{\prime}$ denotes $d F_{I}\left(T_{-}\right) / d T_{-}$. We need a regularized source $S_{44}^{\text {reg }} \sim O\left(r^{-2}\right)$ at least [i.e., the same as the potential $V_{Z} \sim$ $\left.O\left(r^{-2}\right)\right]$. At the horizon the source behaves well, $S_{44} \sim$ $O[(r-2)]$, with

$$
\psi_{22}^{(1)}=F_{H}^{\prime}+\frac{1}{4} F_{H}+\frac{27}{56}(r-2) F_{H}+O\left[(r-2)^{2}\right],
$$

where $F_{H}=F_{H}\left(T_{+}\right)$is some function of $T_{+}=t+r_{*}$.

We can regularize the source term by using the regularized function,

$$
\chi_{44}^{(2) \mathrm{reg}}=\chi_{44}^{(2)}-\frac{\sqrt{70}}{126 \sqrt{\pi}} \frac{(r-2)^{2}}{r} \frac{\partial \psi_{22}^{(1)}}{\partial r} \frac{\partial^{2} \psi_{22}^{(1)}}{\partial r \partial t},
$$


which satisfies the Zerilli Eq. (9) with a well-behaved source term, $S_{44}^{\text {reg }} \sim O\left(r^{-2}\right)$ at infinity and $S_{44}^{\text {reg }} \sim O[(r-$ $2)]$ at the horizon. Thus we can remove an unphysical gauge-dependent divergence. Note that such a regularization is not unique, and, for example, we can replace $\partial / \partial r$ with $\partial / \partial t$ in Eq. (12). The regularization is equivalent to adding quadratic terms of the first-order gauge invariant function to the second-order gauge invariant function, so that it preserves the gauge invariance [19].

The explicit form of the regularized source term is

$$
\begin{aligned}
S_{44}^{\mathrm{reg}}(t, r)= & \frac{r-2}{42} \frac{\sqrt{70}}{\sqrt{\pi}}\left\{\frac{228 r^{7}+8 r^{6}-370 r^{5}+142 r^{4}-384 r^{3}-514 r^{2}-273 r-48}{r^{5}(3 r+1)^{2}(2 r+3)^{2}} \dot{\psi}^{\prime} \psi^{\prime}\right. \\
& -\frac{72 r^{8}+3936 r^{7}+2316 r^{6}-2030 r^{5}-7744 r^{4}-9512 r^{3}-3540 r^{2}-1119 r-144}{r^{6}(3 r+1)^{2}(2 r+3)^{3}} \psi^{\prime} \dot{\psi} \\
& +\frac{(7 r+4) r}{3(r-2)^{2}} \ddot{\psi} \ddot{\psi}+\frac{24 r^{7}+344 r^{6}-872 r^{5}-771 r^{4}+120 r^{3}+77 r^{2}-237 r-48}{r^{3}(3 r+1)^{2}(2 r+3)^{2}(r-2)^{2}} \ddot{\psi} \dot{\psi} \\
& -\frac{66 r^{4}-106 r^{3}-220 r^{2}-156 r-45}{r(3 r+1)^{2}(2 r+3)(r-2)} \ddot{\psi}^{\prime} \dot{\psi}+\frac{198 r^{5}-318 r^{4}-664 r^{3}-458 r^{2}-127 r-24}{3 r^{2}(3 r+1)^{2}(2 r+3)(r-2)} \dddot{\psi} \psi^{\prime} \\
& -\frac{3\left(2160 r^{9}+11760 r^{8}+30560 r^{7}+41124 r^{6}+31596 r^{5}+11630 r^{4}-1296 r^{3}-4182 r^{2}-1341 r-144\right)}{r^{7}(2 r+3)^{4}(3 r+1)^{2}} \psi \dot{\psi} \\
& -\frac{7 r+4}{3 r} \ddot{\psi}^{\prime} \dot{\psi}^{\prime}-\frac{2(r-2)}{3(3 r+1)^{2} r^{2}} \ddot{\psi} \dot{\psi}^{\prime}+\frac{252 r^{6}+636 r^{5}+674 r^{4}+730 r^{3}+524 r^{2}+171 r+24}{r^{3}(3 r+1)^{2}(2 r+3)^{2}(r-2)} \psi \ddot{\psi} \\
& \left.-\frac{216 r^{8}+4296 r^{7}+1992 r^{6}-3488 r^{5}-8716 r^{4}-9512 r^{3}-3540 r^{2}-1119 r-144}{r^{6}(3 r+1)^{2}(2 r+3)^{3}} \psi \dot{\psi}^{\prime}\right\},
\end{aligned}
$$

where $\dot{\psi}=\partial \psi_{22}^{(1)} / \partial t$ and $\psi^{\prime}=\partial \psi_{22}^{(1)} / \partial r$. We can now solve the regularized Zerilli Eq. (9). With Fourier expansions, this provides a two-point boundary value problem with purely ingoing boundary condition at the horizon and purely outgoing at infinity. The numerical calculation will be presented in a forthcoming paper.

\section{DETECTABILITY}

Without solving Eq. (9) numerically, we can find the essential properties of the solutions, i.e., the QNM frequency $\omega_{44 n}^{(2)}$ and the order-of-magnitude QNM amplitude. Since the source term $S_{44}^{(2) \text { reg }}$ is quadratic in the first-order function $\psi_{22}^{(1)} \propto e^{-i \omega_{22 n}^{(1)} t}$, we have $\chi_{44}^{(2) \text { reg }} \propto e^{-2 i \omega_{22 n}^{(1)} t}$ from Eq. (9). Therefore the second-order QNM frequencies are twice the first-order ones,

$$
\omega_{44 n}^{(2)}=2 \omega_{22 n}^{(1)},
$$

which differ from any first-order QNM frequencies $\omega_{\ell m n}^{(1)}$. By matching the dimensions in both sides of Eq. (9), we also estimate the order-of-magnitude amplitude as

$$
\left|\chi_{44}^{(2) \text { reg }}\right| \sim\left|\omega_{22 n}^{(1)} \psi_{22}^{(1)}\right|^{2} .
$$

Then we can derive the second-order QNM energy $E^{(2)}$ from the first-order one $E^{(1)}$. For the QNM waveform, $\psi_{22}^{(1)}=\psi_{22}^{(1)}(0) e^{-i \omega_{22 n}^{(1)} t}$, the first-order energy $E^{(1)}$ in Eq. (7) is given by

$$
\frac{E^{(1)}}{M}=\frac{3}{8 \pi} \frac{\left|\omega_{22 n}^{(1)}\right|^{2}\left|\psi_{22}^{(1)}(0)\right|^{2}}{M\left|\Im \omega_{22 n}^{(1)}\right|},
$$

where $E^{(1)} / M \sim 1 \%$ for equal-mass mergers [6]. By noting $\chi_{44}^{(2)} \sim \partial \psi_{44}^{(2)} / \partial t \sim 2 \omega_{22 n}^{(1)} \psi_{44}^{(2)}$, we have a similar expression for the second-order energy $E^{(2)}$ as

$$
\frac{E^{(2)}}{M} \sim \frac{45}{8 \pi} \frac{\left|\chi_{44}^{(2) \operatorname{reg}}(0)\right|^{2}}{M\left|2 \Im \omega_{22 n}^{(1)}\right|} \sim M\left|\Im \omega_{22 n}^{(1)}\right|\left(\frac{E^{(1)}}{M}\right)^{2},
$$

where the second equality uses Eqs. (15) and (16). In a future paper we will calculate the coefficient as $E^{(2)} / M \sim$ $15 M\left|\Im \omega_{22 n}^{(1)}\right|\left(E^{(1)} / M\right)^{2}$.

Once we know the QNM frequency $\omega$ and energy $E$, we can obtain the GW energy spectrum $[3,20]$

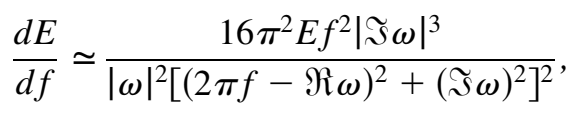

and then the characteristic amplitude $h_{\text {char }}(f)$ with Eq. (5.1) of Flanagan and Hughes [3],

$$
h_{\text {char }}(f)^{2}=\frac{2(1+z)^{2}}{\pi^{2} D(z)^{2}} \frac{d E}{d f}[(1+z) f],
$$

where $D(z)$ is the luminosity distance.

\section{DISCUSSIONS}

Our main results are summarized in Fig. 1 showing the characteristic GW amplitudes $h_{\text {char }}(f)$ of the Schwarz- 
schild BH QNMs for two equal-mass binary BH mergers of total mass $10^{6} M_{\odot}$ at redshift $z=5$, together with the rms noise amplitude $h_{n}(f)=\sqrt{5 f S_{h}(f)}$ for the space-based detector LISA [3,20] and ultimate DECIGO [1]. The first-order QNM has $\ell=2, m=2$ and energy $E^{(1)} / M=$ $1 \%$. We estimate the second-order QNM with Eqs. (17)(19) and the third-order QNM energy by extrapolating the second-order Eq. (17) as $E^{(3)} / M \sim 15(M \Im \omega)^{2}\left(E^{(1)} / M\right)^{3}$. We can see that the second- and third-order QNMs appear at 2 and 3 times the first-order frequency, respectively, with detectable amplitudes.

We can in principle identify the higher-order QNMs since their frequencies differ from any first-order ones. However the actual identification depends on the SNR of the observations [20] or the accuracy of the simulations. In simulations with current accuracy we may have already mistaken the second-order QNMs for the first-order ones. For example $2 M \omega_{2 m 0}^{(1)}=0.7473-0.1779 i$ is close to $M \omega_{4 m 0}^{(1)}=0.80918-0.09416 i[16]$.

In order to prove that the second-order QNMs actually exist, we have to find the second-order QNMs directly in the numerical simulations. Such simulations are challenging because the mesh size should be less than $\sim 1 \% \times M$ to resolve $\sim 1 \%$ metric perturbations. Even $1+1 \mathrm{D}$ spherical models for the fully self-gravitating case have not found the second-order QNMs [21]. Simulations of acoustic black holes may be an alternative for this purpose [22]. We also need a mathematically rigorous definition of second-order QNMs like the first-order ones that use the Laplace transformation rather than the Fourier transformation $[2,23]$.

Future problems include the Kerr $\mathrm{BH}$ case, the odd parity mode case, and a more solid third-order formulation. Since the master equation for Kerr BHs also has a source term quadratic in the first-order function [12], we may expect similar results. When BHs have no spin before mergers, the final spin is $a \sim 0.7$ [6] and hence the Kerr effects may not be so large (as inferred from the fact that the QNM frequencies shift by only a small factor). The odd parity mode appears when BHs have spin before mergers. At the third-order the QNM frequencies will also shift up to $\left(\psi^{(1)} / M\right)^{2} \sim 1 \%$ as suggested by the anharmonic oscillations in [8], probably blueward because the GW carries away the $\mathrm{BH}$ mass.

The ratio between the first- and higher-order QNM amplitudes include new information about the total GW

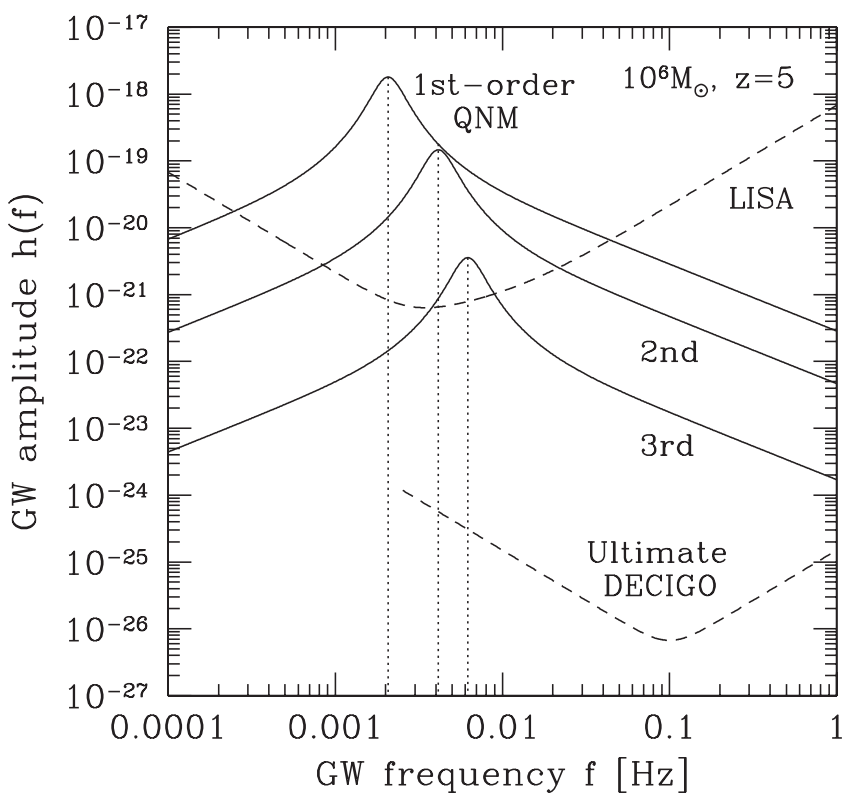

FIG. 1. The characteristic GW amplitudes $h_{\text {char }}(f)$ of the Schwarzschild BH QNMs for two equal-mass binary BH mergers of total mass $10^{6} M_{\odot}$ at redshift $z=5$. The first-order QNM has $\ell=2, m=2$ and energy $E^{(1)} / M=1 \%$. The second- and third-order QNMs also appear at 2 and 3 times the first-order frequency, respectively, with detectable amplitudes. The dashed line is the rms noise amplitude $h_{n}(f)=\sqrt{5 f S_{h}(f)}$ for the spacebased detector LISA and ultimate DECIGO. The signal-to-noise ratio squared for a randomly oriented source is given by $(\mathrm{SNR})^{2}=\int d(\ln f)\left[h_{\text {char }}(f) / h_{n}(f)\right]^{2}$. We use a cosmology $\left(\Omega_{m}, \Omega_{\Lambda}, h\right)=(0.27,0.73,0.71)$. We estimate the third-order QNM energy by extrapolating the second-order Eq. (17) as $E^{(3)} / M \sim 15(M \Im \omega)^{2}\left(E^{(1)} / M\right)^{3}$.

energy $E$. Since the observed GW amplitude is $h \sim$ $(E / M)^{1 / 2}(M / r)$, this could provide a distance indicator.

\section{ACKNOWLEDGMENTS}

We thank C. O. Lousto and Y. Zlochower for a careful reading of the manuscript. This work is supported in part by the Grant-in-Aid (18740147) from the Ministry of Education, Culture, Sports, Science, and Technology (MEXT) of Japan (K. I.). This is also supported by the Japan Society for the Promotion of Science for Research Abroad and in part by the NSF for financial support from Grant No. PHY-0722315 (H. N.).
[1] N. Seto, S. Kawamura, and T. Nakamura, Phys. Rev. Lett. 87, 221103 (2001).

[2] K. D. Kokkotas and B. G. Schmidt, Living Rev. Relativity 2, 2 (1999)
[3] E. E. Flanagan and S. A. Hughes, Phys. Rev. D 57, 4535 (1998).

[4] M. Enoki, K. T. Inoue, M. Nagashima, and N. Sugiyama, Astrophys. J. 615, 19 (2004). 
[5] K. Ioka and P. Meszaros, Astrophys. J. 635, 143 (2005).

[6] F. Pretorius, Phys. Rev. Lett. 95, 121101 (2005); M. Campanelli, C.O. Lousto, P. Marronetti, and Y. Zlochower, Phys. Rev. Lett. 96, 111101 (2006); J. G. Baker, J. Centrella, D. I. Choi, M. Koppitz, and J. van Meter, Phys. Rev. Lett. 96, 111102 (2006).

[7] E. Berti, V. Cardoso, J. A. Gonzalez, U. Sperhake, M. Hannam, S. Husa, and B. Bruegmann, arXiv:gr-qc/ 0703053.

[8] L. D. Landau and E. M. Lifshitz, Mechanics (Butterworth, Heinemann, Oxford, 1976), 3rd ed..

[9] K. Tomita, Prog. Theor. Phys. 52, 1188 (1974); K. Tomita and N. Tajima, Prog. Theor. Phys. 56, 551 (1976).

[10] R. J. Gleiser, C. O. Nicasio, R. H. Price, and J. Pullin, Classical Quantum Gravity 13, L117 (1996); Phys. Rev. Lett. 77, 4483 (1996); Phys. Rep. 325, 41 (2000); C. O. Nicasio, R. Gleiser, and J. Pullin, Gen. Relativ. Gravit. 32, 2021 (2000).

[11] V. Acquaviva, N. Bartolo, S. Matarrese, and A. Riotto, Nucl. Phys. B667, 119 (2003); S. Matarrese, S. Mollerach, and M. Bruni, Phys. Rev. D 58, 043504 (1998); K. Tomita, Phys. Rev. D 71, 083504 (2005); K. Nakamura, Prog. Theor. Phys. 117, 17 (2007).
[12] M. Campanelli and C. O. Lousto, Phys. Rev. D 59, 124022 (1999).

[13] M. Bruni, S. Matarrese, S. Mollerach, and S. Sonego, Classical Quantum Gravity 14, 2585 (1997); K. Nakamura, Prog. Theor. Phys. 110, 723 (2003); D. Brizuela, J. M. Martin-Garcia, and G. A. M. Marugan, J. Phys. Conf. Ser. 66, 012011 (2007).

[14] T. Regge and J. Wheeler, Phys. Rev. 108, 1063 (1957).

[15] F. J. Zerilli, Phys. Rev. D 2, 2141 (1970).

[16] E. W. Leaver, Proc. R. Soc. A 402, 285 (1985).

[17] N. Sago, H. Nakano, and M. Sasaki, Phys. Rev. D 67, 104017 (2003).

[18] K. Martel and E. Poisson, Phys. Rev. D 71, 104003 (2005).

[19] A. Garat and R.H. Price, Phys. Rev. D 61, 044006 (2000).

[20] E. Berti, V. Cardoso, and C. M. Will, Phys. Rev. D 73, 064030 (2006).

[21] Y. Zlochower, R. Gomez, S. Husa, L. Lehner, and J. Winicour, Phys. Rev. D 68, 084014 (2003).

[22] S. Okuzumi and M. a. Sakagami, arXiv:gr-qc/0703070.

[23] E. W. Leaver, Phys. Rev. D 34, 384 (1986). 